\title{
An Unprovoked Haemolytic-Uremic Syndrome; A Unique Case Report
}

\author{
Fateme Sadat Tabatabaei ${ }^{1}$, Maryam Moghaddam ${ }^{1}$, Farshid Kompani ${ }^{2}$ and Seyyed Mohsen Hosseininejad*3,4,5 \\ ${ }^{1}$ Pediatric resident, Neonatal and Children's Health Research Center, Golestan University of Medical Sciences, Iran \\ ${ }^{2}$ Assistant professor of pediatric nephrology, Golestan University of Medical Sciences, Iran \\ ${ }^{3}$ Neonatal and Children's Health Research Center, Golestan University of Medical Sciences, Gorgan, Iran \\ ${ }^{4}$ Student Research Committee, Shahid Beheshti University of Medical Sciences, Tehran, Iran \\ ${ }^{5}$ North Khorasan University of Medical Sciences, Bojnurd, Iran
}

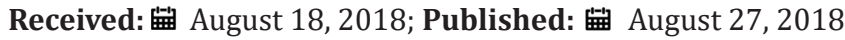

*Corresponding author: Seyyed Mohsen Hosseininejad, Shastkola, Student Research Committee, Golestan University of Medical Sciences, Gorgan, Iran

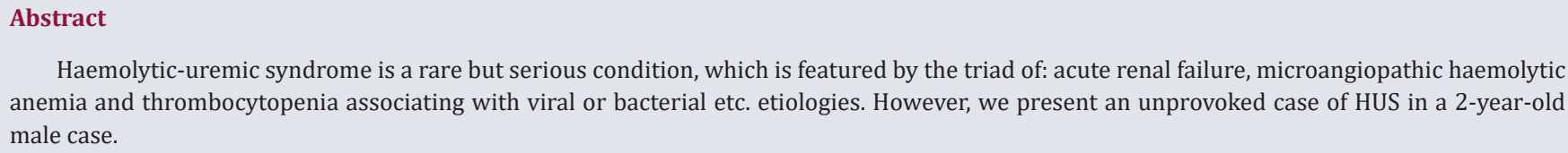

Haemolytic-uremic syndrome is a rare but serious condition, which is featured by the triad of: acute renal failure, microangiopathic haemolytic anemia and thrombocytopenia associating with viral or bacterial etc. etiologies. However, we present an unprovoked case of HUS in a 2-year-old male case.

Keywords: Case Report, Hemolytic-Uremic Syndrome

\section{Introduction}

Hemolytic Uremic Syndrome (HUS) is a rare life-threatening disorder. It is recognized by hemolysis, thrombocytopenia, and acute renal failure. Organs comprising the brain, intestines, pancreas, heart, and lungs might be influenced and damaged during the condition, [1-4]. It is frequently precipitated by gastroenteritis but provoked by bacterial or viral infection especially in pediatric cases. An etiological review of previous reported cases with HUS will be given in the following as well [3-5]. Even if we dedicated attention on HUS risk related with bacterial/viral infections, we identified a 2-year-old male baby compatible with HUS features, most probably without any potential known endo/exogenous triggers whose interesting report will be narrated as below.

\section{Case Presentation}

We are reporting the case of a 2-year-old male Caucasian baby who was hospitalized with 8 days history acute severe watery diarrhea gradually reduced into a bloody low-volume diarrhea, accompanied with vomiting, intermittent fever and weakened general condition but no coriza symptoms; unresponsive to home-administered medication-ibuprofen, acetaminophen except for fever. He was born from a consanguineous marriage with no significant past medical history. At the time of admission, his physical examination revealed pale skin, dry lips, decreased skin turgor, no pharyngeal hyperemia, no budging fontanel, respiratory rate of 28 breaths per minute, blood pressure (BP): 67 / 50mmHg, heart rate (HR) of 122 beats per minute (bpm), food refusal, and bloody stools. No lymphadenopathy nor neurologic sings were detected neither. Laboratory tests showed the following results as: leukocytosis: 12400 (Poly: 65\%), hemoglobin: 9.2g/L, hematocrit 32\%, BUN: $128 \mathrm{mg} / \mathrm{dl}, \mathrm{Cr}: 4.4 \mathrm{mg} / \mathrm{dl}$ and Normal hepatic tests on admission. In progress: persistent leukocytosis of 20430 with Poly of $63 \%$, decreasing anemia $9.2-10.1 \mathrm{~g} / \mathrm{L}$, lactate dehydrogenase $5834 \mathrm{U} / \mathrm{L}$ (references value $=615 \mathrm{U} / \mathrm{L}$ ), changes indicative for an acute renal failure-blood urea nitrogen 128 to $154 \mathrm{mg} / \mathrm{dL}$, creatinine 4.10 to $4.72 \mathrm{mg} / \mathrm{dL}$, hepatocytolysis, gamma-glutamyl transferase (gGT) 135U/L(references value 0-39U/L), and a C-reactive protein of $17 \mathrm{mg} / \mathrm{L}$ (references value $0-10 \mathrm{mg} / \mathrm{L}$ ). The complement tests and ANA and anti-ds DNA were: C3: 0.68, C4: 0.11, CH50: 16; Anti-ds DNA: 3.4, ANA: 1.2.his hepatitis Ag tests and ant phospholipid tests were negative. His peripheral blood smear showed poikilocytosis, anisocytosis, and schizocytes.

Stool examinations for bacterial organisms or relevant antigens was not positive for Salmonella, Shigella, Campylobacter, Yersinia, E coli 0157, enterohemorrhagic E coli; virulence molecular markers for verocytotoxin-producing E coli (VTEC/vtx1, vtx2), enteropathogenic E coli (EPEC/eae), enterotoxigenic E coli (ETEC elt, est) and enteroinvasive E coli (EIEC/ipaH) were also negative. Repeated check of stool sample for viruses including rotavirus, adenovirus and norovirus were done; this was also unremarkable. Other systemic examinations and imagings were negative for any pathologic evidence as well. while hospitalization for appropriate monitoring of urine output, catheterization was implemented, 
observing oligoanuria with a volume of $12 \mathrm{~mL}$ urine/24 hours under treatment with serum mannitol and lasix. Naturally, there was an augmented nitrogen retention, and anemia related with thrombocytopenia which was improved with tight control of hydration. The patient needed cardiovascular watching one month after his discharge. His renal function became adjusted on discharge.

\section{Discussion}

HUS in pediatric is mainly triggered by STEC 0157, followed by 026. Since 2010, it was told that 2350 cases initiated by STEC 026 till 2014, [5-7] with a peak in 2015, respectively, 463 cases. In the United States, the incidence of HUS in children under six years is of $6.1 / 100,000$ population/year. In 2012, 274 cases associated with diarrhea were described $[1,8]$. Other potential etiologies of HUS are: other bacterial infections (Salmonella, Shigella dysenteriae, Campylobacter, Streptococcus pneumoniae, Mycoplasma, Legionella, etc.), viral infections (adenoviruses, enteroviruses, HIV, Epstein Barr virus, herpes simplex, Portillo virus, etc.), but there are also noninfectious causes: as the advance of a neoplasia (pancreatic, gastric, and prostate cancer), drug-induced (anticancer medication, quinine, antiplatelet drugs, contraceptives), post-transplantation (bone marrow, lung, kidney, etc.) and during pregnancy [9] or linked with the antiphospholipid syndrome, systemic lupus erythematosus or hierarchy origins with autosomal dominant or recessive heritage.

Regarding pathophysiology, HUS is the result of endothelial damage, ordinarily being an outcome of the effect of Shiga toxin which attaches to the cell membrane receptor, $[1,10]$ and other infectious or noninfectious triggers, with the release of vasoactive affluences, glomerular microangiopathy and of small renal arterioles [11]. Acute renal failure in the advancement of HUS is correctable in $85 \%$ of cases under supportive care. The risk factors for the development into HUS are the age of more the five years old, different etiologies from STEC, insistent oligoanuria, glomerular impairment and central nervous system (80\%). The combined leukocytosis and fever characterize an augmented threat for Hemolytic uremic syndrome. HUS is blamable for seven percentage of cases with hypertension in infants, being the principal cause in chronic renal derangement in pediatric, and a main cause of important kidney injury in adults. This current case is unique as no established potential etiology was found for HUS with occurrence of loose stools which could have masked diminished urine output and resulted in acute renal failure, linking other harsh prognostic features such as leukocytosis, fever and non-STEC etiology. Observing renal function, blood pressure, and heart rate will be obligatory afterward.

\section{Conclusion}

Although the most common etiology of HUS remains STEC, other etiologies like viral causes should not be neglected, keeping in mind the fact that there might be further unrecognized etiologies responsible for developing HUS.

\section{Declaration}

An informed consent was obtained from patients' parents; there is no conflict of interest regarding submission of manuscript.

\section{Acknowledgment}

The authors wish to express their sincere gratitude to our patient's family for their patience and cooperation. We are also grateful to nursing staff of Talrghani Pediatric Hospital, Gorgan, Northern Iran.

\section{References}

1. Keir LS (2015) Shiga toxin associated hemolytic uremic syndrome. Hematol Oncol Clin North Am29(3): 525-539.

2. Conway EM (2015) HUS and the case for complement. Blood 126(18): 2085-2090.

3. Siegler RL (1988) Management of hemolytic-uremic syndrome. J Pediatr 112(6): 1014-1020.

4. Siegler R, Oakes R (2005) Hemolytic uremic syndrome; pathogenesis, treatment, and outcome. Curr Opin Pediatr 17(2): 200-204.

5. Peron E, Zaharia A, Zota L, Severi E, Mardh O, et al. (2016) Early findings in outbreak of haemolytic uraemic syndrome among young children caused by Shigatoxin-producing Escherichia coli, Romania, January to February 2016. Euro Surveill 21(11): 30170.

6. (2016) European Centre for Disease Prevention and Control/European Food Safety Authority. Multicountry outbreak of STEC infection associated with HUS. Stockholm: ECDC 2016.

7. (2015) FSA (European Food Safety Authority) and ECDC (European Centre for Disease Prevention and Control) The European Union summary report on trends and sources of zoonoses, zoonotic agents and food borne outbreaks in 2016 EFSA J 13: 4329.

8. Adams DA, Jajosky RA, Ajani U, Jeffrey K, Pearl S, et al. (2014) Summary of notifiable diseases-United States, 2012. MMWR Morb Mortal Wkly Rep 61(53): 1-21.

9. Saad AF, Roman J, Wyble A, Luis DP (2016) Pregnancy-associated atypical hemolytic-uremic syndrome. AJP Rep 6(1): 125-128.

10. Obrig TG, Kaplan BS, Trompeter RS, Moake JL (1992) Pathogenesis of Shiga toxin (verotoxin)-induced endothelial cell injury, in hemolytic uremic syndrome and thrombotic. Thrombocytopenic Purpura Marcel Dekker, New York 6(4): 405-419.

11. Gianviti A, Tozzi AE, De Petris L, Alfredo C, Lucilla R, et al. (2003) Risk factors for poor renal prognosis in children with hemolytic uremic syndrome. Pediatr Nephrol 18(12) :1229-1235. 
ISSN: 2574-1241

DOI: $10.26717 / B J S T R .2018 .08 .001647$

Seyyed Mohsen Hosseininejad. Biomed J Sci \& Tech Res

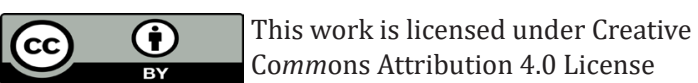

Submission Link: https://biomedres.us/submit-manuscript.php

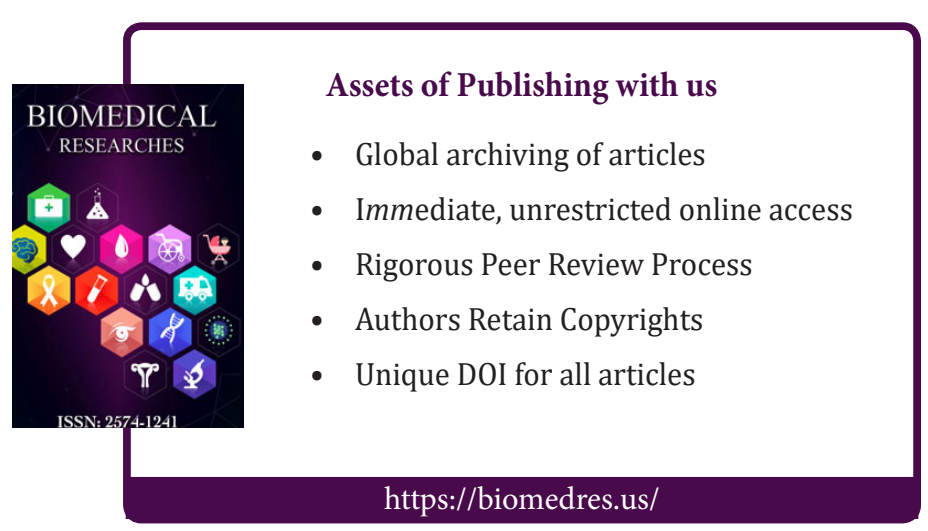

